\title{
ANISOTROPIC GEVREY-HÖRMANDER PSEUDO-DIFFERENTIAL OPERATORS ON MODULATION SPACES
}

\author{
AHMED ABDELJAWAD AND JOACHIM TOFT
}

\begin{abstract}
We show continuity properties for the pseudo-differential operator $\operatorname{Op}(a)$ from $M\left(\omega_{0} \omega, \mathscr{B}\right)$ to $M(\omega, \mathscr{B})$, for fixed $s, \sigma \geq 1$, $\omega, \omega_{0} \in \mathscr{P}_{s, \sigma}^{0}\left(\omega, \omega_{0} \in \mathscr{P}_{s, \sigma}\right), a \in \Gamma_{\left(\omega_{0}\right)}^{\sigma, s}\left(a \in \Gamma_{\left(\omega_{0}\right)}^{\sigma, s ; 0}\right)$, and $\mathscr{B}$ is an invariant Banach function space.
\end{abstract}

\section{INTRODUCTION}

In the paper, we consider pseudo-differential operators, where the symbols are of infinite orders and possess suitable Gevrey regularities and which are allowed to grow sub-exponentially together with all their derivatives. Our main purpose is to extend boundedness results, in [38], of the pseudo-differential operators when acting on modulation spaces.

More specific, the symbols should satisfy conditions of the form

$$
\left|\partial_{x}^{\alpha} \partial_{\xi}^{\beta} a(x, \xi)\right| \lesssim h^{|\alpha+\beta|} \alpha !^{\sigma} \beta !^{s} \omega_{0}(x, \xi),
$$

where $\omega_{0}$ should be a moderate weight on $\mathbf{R}^{2 d}$ and satisfy boundedness conditions like

$$
\omega_{0}(x, \xi) \lesssim e^{r\left(|x|^{\frac{1}{s}}+|\xi|^{\frac{1}{\sigma}}\right)}
$$

For such symbols $a$ we prove that corresponding pseudo-differential operators $\operatorname{Op}(a)$ is continuous from the modulation space $M\left(\omega_{0} \omega, \mathscr{B}\right)$ to $M(\omega, \mathscr{B})$. (See Section 1 for notations.)

Similar investigations were performed in [43] in the case $s=\sigma$ (i.e. the isotropic case). Therefore, the results in the current paper are more general in the sense of the anisotropicity of the considered symbol classes. Moreover, we use different techniques compared to [43].

We also remark that several ideas arise in [38], where similar investigations were performed after the conditions (0.1) and (0.2) are replaced by

and

$$
\left|\partial_{x}^{\alpha} \partial_{\xi}^{\beta} a(x, \xi)\right| \lesssim \omega_{0}(x, \xi)
$$

$$
\omega_{0}(x, \xi) \lesssim(1+|x|+|\xi|)^{N}
$$

respectively, for some $N \geq 0$.

2010 Mathematics Subject Classification. 35S05, 47B37, 47G30, 42B35.

Key words and phrases. Pseudo-differential operators, Modulation spaces, Banach function spaces, Gelfand-Shilov spaces, Gevrey regularity, 
In [11, H. Feichtinger introduced the modulation spaces to measure the time-frequency concentration of a function or distribution on the time-frequency space or the phase space $\mathbf{R}^{2 d}$. Nowadays they become popular among mathematicians and engineers since their numerous applications in signal processing [13, 14], pseudo-differential and Fourier integral operators [4-6, 30, 31, 35, 43] and quantum mechanics [7, 20].

The paper is organized as follows. In Section 1 we give the main definition and properties of Gelfand-Shilov and modulation spaces and we recall some essential results. In Section 2 we state our main results on the continuity with anisotropic settings.

\section{Preliminaries}

In the current section we review basic properties for modulation spaces and other related spaces. More details and proofs can be found in [9 11, 15 18, 22, 40].

1.1. Weight functions. A function $\omega$ on $\mathbf{R}^{d}$ is called a weight or weight function, if $\omega, 1 / \omega \in L_{\mathrm{loc}}^{\infty}\left(\mathbf{R}^{d}\right)$ are positive everywhere. The weight $\omega$ on $\mathbf{R}^{d}$ is called $v$-moderate for some weight $v$ on $\mathbf{R}^{d}$, if

$$
\omega(x+y) \lesssim \omega(x) v(y), \quad x, y \in \mathbf{R}^{d} .
$$

If $v$ is even and satisfies (1.1) with $\omega=v$, then $v$ is called submultiplicative.

Let $s, \sigma>0$. Then we let $\mathscr{P}_{E}\left(\mathbf{R}^{d}\right)$ be the set of all moderate weights on $\mathbf{R}^{d}, \mathscr{P}_{s}\left(\mathbf{R}^{d}\right)\left(\mathscr{P}_{s}^{0}\left(\mathbf{R}^{d}\right)\right)$ be the set of all $\omega \in \mathscr{P}_{E}\left(\mathbf{R}^{d}\right)$ such that

$$
\omega(x+y) \lesssim \omega(x) e^{r|y|^{\frac{1}{s}}}, \quad x, y \in \mathbf{R}^{d},
$$

for some $r>0$ (for every $r>0$ ), and $\mathscr{P}_{s, \sigma}\left(\mathbf{R}^{2 d}\right)\left(\mathscr{P}_{s, \sigma}^{0}\left(\mathbf{R}^{2 d}\right)\right)$ be the set of all $\omega \in \mathscr{P}_{E}\left(\mathbf{R}^{2 d}\right)$ such that

$$
\omega(x+y, \xi+\eta) \lesssim \omega(x, \xi) e^{r\left(|y|^{\frac{1}{s}}+|\eta|^{\frac{1}{\sigma}}\right)}, \quad x, y, \xi, \eta \in \mathbf{R}^{d},
$$

for some $r>0$ (for every $r>0$ ).

The following result shows that for any weight in $\mathscr{P}_{E}$, there are equivalent weights that satisfy strong Gevrey regularity.

Proposition 1.1. Let $\omega \in \mathscr{P}_{E}\left(\mathbf{R}^{2 d}\right)$ and $s, \sigma>0$. Then there exists a weight $\omega_{0} \in \mathscr{P}_{E}\left(\mathbf{R}^{2 d}\right) \cap C^{\infty}\left(\mathbf{R}^{2 d}\right)$ such that the following is true:

(1) $\omega_{0} \asymp \omega$;

(2) for every $h>0$,

$$
\left|\partial_{x}^{\alpha} \partial_{\xi}^{\beta} \omega_{0}(x, \xi)\right| \lesssim h^{|\alpha+\beta|} \alpha !^{\sigma} \beta !^{s} \omega_{0}(x, \xi) \asymp h^{|\alpha+\beta|} \alpha !^{\sigma} \beta !^{s} \omega(x, \xi) .
$$

Proposition 1.1] is equivalent to [2, Proposition 1.6]. In fact, by Proposition [2, Proposition 1.6] we have that Proposition 1.1 holds with $s=\sigma$. Hence, Proposition 1.1 implies [2, Proposition 1.6]. On the 
other hand, let $s_{0}=\min (s, \sigma)$. Then [2, Proposition 1.6] implies that there is a weight function $\omega_{0} \asymp \omega$ satisfying

$$
\begin{aligned}
\left|\partial_{x}^{\alpha} \partial_{\xi}^{\beta} \omega_{0}(x, \xi)\right| & \lesssim h^{|\alpha+\beta|}(\alpha ! \beta !)^{s 0} \omega_{0}(x, \xi) \\
& \lesssim h^{|\alpha+\beta|} \alpha !^{\sigma} \beta !^{s} \omega_{0}(x, \xi),
\end{aligned}
$$

giving Proposition 1.1.

1.2. Gelfand-Shilov spaces. Let $0<h, s, \sigma \in \mathbf{R}$ be fixed. Then $\mathcal{S}_{s ; h}^{\sigma}\left(\mathbf{R}^{d}\right)$ is the Banach space of all $f \in C^{\infty}\left(\mathbf{R}^{d}\right)$ such that

$$
\|f\|_{\mathcal{S}_{s ; h}^{\sigma}} \equiv \sup _{\alpha, \beta \in \mathbf{N}^{d}} \sup _{x \in \mathbf{R}^{d}} \frac{\left|x^{\alpha} \partial^{\beta} f(x)\right|}{h^{|\alpha|+|\beta|} \alpha !^{s} \beta ! \sigma}<\infty,
$$

endowed with the norm (1.3).

The Gelfand-Shilov spaces $\mathcal{S}_{s}^{\sigma}\left(\mathbf{R}^{d}\right)$ and $\Sigma_{s}^{\sigma}\left(\mathbf{R}^{d}\right)$ are defined as the inductive and projective limits respectively of $\mathcal{S}_{s ; h}^{\sigma}\left(\mathbf{R}^{d}\right)$. This implies that

$$
\mathcal{S}_{s}^{\sigma}\left(\mathbf{R}^{d}\right)=\bigcup_{h>0} \mathcal{S}_{s ; h}^{\sigma}\left(\mathbf{R}^{d}\right) \quad \text { and } \quad \Sigma_{s}^{\sigma}\left(\mathbf{R}^{d}\right)=\bigcap_{h>0} \mathcal{S}_{s ; h}^{\sigma}\left(\mathbf{R}^{d}\right),
$$

and that the topology for $\mathcal{S}_{s}^{\sigma}\left(\mathbf{R}^{d}\right)$ is the strongest possible one such that the inclusion map from $\mathcal{S}_{s ; h}^{\sigma}\left(\mathbf{R}^{d}\right)$ to $\mathcal{S}_{s}^{\sigma}\left(\mathbf{R}^{d}\right)$ is continuous, for every choice of $h>0$. The space $\Sigma_{s}^{\sigma}\left(\mathbf{R}^{d}\right)$ is a Fréchet space with seminorms $\|\cdot\|_{\mathcal{S}_{s ; h}^{\sigma}}, h>0$. Moreover, $\Sigma_{s}^{\sigma}\left(\mathbf{R}^{d}\right) \neq\{0\}$, if and only if $s+\sigma \geq 1$ and $(s, \sigma) \neq\left(\frac{1}{2}, \frac{1}{2}\right)$, and $\mathcal{S}_{s}^{\sigma}\left(\mathbf{R}^{d}\right) \neq\{0\}$, if and only if $s+\sigma \geq 1$.

The Gelfand-Shilov distribution spaces $\left(\mathcal{S}_{s}^{\sigma}\right)^{\prime}\left(\mathbf{R}^{d}\right)$ and $\left(\Sigma_{s}^{\sigma}\right)^{\prime}\left(\mathbf{R}^{d}\right)$ are the projective and inductive limit respectively of $\left(\mathcal{S}_{s ; h}^{\sigma}\right)^{\prime}\left(\mathbf{R}^{d}\right)$. In [29] it is proved that $\left(\mathcal{S}_{s}^{\sigma}\right)^{\prime}\left(\mathbf{R}^{d}\right)$ is the dual of $\mathcal{S}_{s}^{\sigma}\left(\mathbf{R}^{d}\right)$, and $\left(\Sigma_{s}^{\sigma}\right)^{\prime}\left(\mathbf{R}^{d}\right)$ is the dual of $\Sigma_{s}^{\sigma}\left(\mathbf{R}^{d}\right)$ (also in topological sense).

The Fourier transform $\mathscr{F}$ is the linear and continuous map on $\mathscr{S}\left(\mathbf{R}^{d}\right)$, given by the formula

$$
(\mathscr{F} f)(\xi)=\widehat{f}(\xi) \equiv(2 \pi)^{-\frac{d}{2}} \int_{\mathbf{R}^{d}} f(x) e^{-i\langle x, \xi\rangle} d x
$$

when $f \in \mathscr{S}\left(\mathbf{R}^{d}\right)$. Here $\langle\cdot, \cdot\rangle$ denotes the usual scalar product on $\mathbf{R}^{d}$. The Fourier transform extends uniquely to homeomorphisms from $\left(\mathcal{S}_{s}^{\sigma}\right)^{\prime}\left(\mathbf{R}^{d}\right)$ to $\left(\mathcal{S}_{\sigma}^{s}\right)^{\prime}\left(\mathbf{R}^{d}\right)$, and from $\left(\Sigma_{s}^{\sigma}\right)^{\prime}\left(\mathbf{R}^{d}\right)$ to $\left(\Sigma_{\sigma}^{s}\right)^{\prime}\left(\mathbf{R}^{d}\right)$. Furthermore, it restricts to homeomorphisms from $\mathcal{S}_{s}^{\sigma}\left(\mathbf{R}^{d}\right)$ to $\mathcal{S}_{\sigma}^{s}\left(\mathbf{R}^{d}\right)$, and from $\Sigma_{s}^{\sigma}\left(\mathbf{R}^{d}\right)$ to $\Sigma_{\sigma}^{s}\left(\mathbf{R}^{d}\right)$.

Some considerations later on involve a broader family of GelfandShilov spaces. More precisely, for $s_{j}, \sigma_{j} \in \mathbf{R}_{+}, j=1,2$, the GelfandShilov spaces $\mathcal{S}_{s_{1}, s_{2}}^{\sigma_{1}, \sigma_{2}}\left(\mathbf{R}^{d_{1}+d_{2}}\right)$ and $\sum_{s_{1}, s_{2}}^{\sigma_{1}, \sigma_{2}}\left(\mathbf{R}^{d_{1}+d_{2}}\right)$ consist of all functions $F \in C^{\infty}\left(\mathbf{R}^{d_{1}+d_{2}}\right)$ such that

$$
\left|x_{1}^{\alpha_{1}} x_{2}^{\alpha_{2}} \partial_{x_{1}}^{\beta_{1}} \partial_{x_{2}}^{\beta_{2}} F\left(x_{1}, x_{2}\right)\right| \lesssim h_{3}^{\left|\alpha_{1}+\alpha_{2}+\beta_{1}+\beta_{2}\right|} \alpha_{1} !^{s_{1}} \alpha_{2} !^{s_{2}} \beta_{1} !^{\sigma_{1}} \beta_{2} !^{\sigma_{2}}
$$


for some $h>0$ respective for every $h>0$. The topologies, and the duals

$$
\left(\mathcal{S}_{s_{1}, s_{2}}^{\sigma_{1}, \sigma_{2}}\right)^{\prime}\left(\mathbf{R}^{d_{1}+d_{2}}\right) \quad \text { and } \quad\left(\Sigma_{s_{1}, s_{2}}^{\sigma_{1}, \sigma_{2}}\right)^{\prime}\left(\mathbf{R}^{d_{1}+d_{2}}\right)
$$

of

$$
\mathcal{S}_{s_{1}, s_{2}}^{\sigma_{1}, \sigma_{2}}\left(\mathbf{R}^{d_{1}+d_{2}}\right) \quad \text { and } \quad \sum_{s_{1}, s_{2}}^{\sigma_{1}, \sigma_{2}}\left(\mathbf{R}^{d_{1}+d_{2}}\right)
$$

respectively, and their topologies are defined in analogous ways as for the spaces $\mathcal{S}_{s}^{\sigma}\left(\mathbf{R}^{d}\right)$ and $\Sigma_{s}^{\sigma}\left(\mathbf{R}^{d}\right)$ above.

The following proposition explains mapping properties of partial Fourier transforms on Gelfand-Shilov spaces, and follows by similar arguments as in analogous situations in [19]. The proof is therefore omitted. Here, $\mathscr{F}_{1} F$ and $\mathscr{F}_{2} F$ are the partial Fourier transforms of $F\left(x_{1}, x_{2}\right)$ with respect to $x_{1} \in \mathbf{R}^{d_{1}}$ and $x_{2} \in \mathbf{R}^{d_{2}}$, respectively.

Proposition 1.2. Let $s_{j}, \sigma_{j}>0, j=1,2$. Then the following is true:

(1) the mappings $\mathscr{F}_{1}$ and $\mathscr{F}_{2}$ on $\mathscr{S}\left(\mathbf{R}^{d_{1}+d_{2}}\right)$ restrict to homeomorphisms

$$
\mathscr{F}_{1}: \mathcal{S}_{s_{1}, s_{2}}^{\sigma_{1}, \sigma_{2}}\left(\mathbf{R}^{d_{1}+d_{2}}\right) \rightarrow \mathcal{S}_{\sigma_{1}, s_{2}}^{s_{1}, \sigma_{2}}\left(\mathbf{R}^{d_{1}+d_{2}}\right)
$$

and

$$
\mathscr{F}_{2}: \mathcal{S}_{s_{1}, s_{2}}^{\sigma_{1}, \sigma_{2}}\left(\mathbf{R}^{d_{1}+d_{2}}\right) \rightarrow \mathcal{S}_{s_{1}, \sigma_{2}}^{\sigma_{1}, s_{2}}\left(\mathbf{R}^{d_{1}+d_{2}}\right)
$$

(2) the mappings $\mathscr{F}_{1}$ and $\mathscr{F}_{2}$ on $\mathscr{S}\left(\mathbf{R}^{d_{1}+d_{2}}\right)$ are uniquely extendable to homeomorphisms

$$
\mathscr{F}_{1}:\left(\mathcal{S}_{s_{1}, s_{2}}^{\sigma_{1}, \sigma_{2}}\right)^{\prime}\left(\mathbf{R}^{d_{1}+d_{2}}\right) \rightarrow\left(\mathcal{S}_{\sigma_{1}, s_{2}}^{s_{1}, \sigma_{2}}\right)^{\prime}\left(\mathbf{R}^{d_{1}+d_{2}}\right)
$$

and

$$
\mathscr{F}_{2}:\left(\mathcal{S}_{s_{1}, s_{2}}^{\sigma_{1}, \sigma_{2}}\right)^{\prime}\left(\mathbf{R}^{d_{1}+d_{2}}\right) \rightarrow\left(\mathcal{S}_{s_{1}, \sigma_{2}}^{\sigma_{1}, s_{2}}\right)^{\prime}\left(\mathbf{R}^{d_{1}+d_{2}}\right) .
$$

The same holds true if the $\mathcal{S}_{s_{1}, s_{2}}^{\sigma_{1} \sigma_{2}}$-spaces and their duals are replaced by corresponding $\Sigma_{s_{1}, s_{2}}^{\sigma_{1}, \sigma_{2}}$-spaces and their duals.

The next two results follow from [8]. The proofs are therefore omitted.

Proposition 1.3. Let $s_{j}, \sigma_{j}>0, j=1,2$. Then the following conditions are equivalent.

(1) $F \in \mathcal{S}_{s_{1}, s_{2}}^{\sigma_{1}, \sigma_{2}}\left(\mathbf{R}^{d_{1}+d_{2}}\right) \quad\left(F \in \Sigma_{s_{1}, s_{2}}^{\sigma_{1}, \sigma_{2}}\left(\mathbf{R}^{d_{1}+d_{2}}\right)\right)$;

(2) for some $h>0$ (for every $h>0$ ) it holds

$$
\left|F\left(x_{1}, x_{2}\right)\right| \lesssim e^{-h\left(\left|x_{1}\right|^{\frac{1}{s_{1}}}+\left|x_{2}\right|^{\frac{1}{s_{2}}}\right)} \quad \text { and } \quad\left|\widehat{F}\left(\xi_{1}, \xi_{2}\right)\right| \lesssim e^{-h\left(\left|\xi_{1}\right|^{\frac{1}{\sigma_{1}}}+\left|\xi_{2}\right|^{\frac{1}{\sigma_{2}}}\right)} \text {. }
$$

We notice that if $s_{j}+\sigma_{j}<1$ for some $j=1,2$, then $\mathcal{S}_{s_{1}, s_{2}}^{\sigma_{1}, \sigma_{2}}\left(\mathbf{R}^{d_{1}+d_{2}}\right)$ and $\sum_{s_{1}, s_{2}}^{\sigma_{1}, \sigma_{2}}\left(\mathbf{R}^{d_{1}+d_{2}}\right)$ are equal to the trivial space $\{0\}$. Likewise, if $s_{j}=$

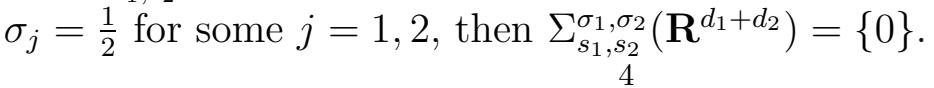




\subsection{Short time Fourier transform and Gelfand-Shilov spaces.}

We recall here some basic facts about the short-time Fourier transform and weights.

Let $\phi \in \mathcal{S}_{s}^{\sigma}\left(\mathbf{R}^{d}\right) \backslash 0$ be fixed. Then the short-time Fourier transform of $f \in\left(\mathcal{S}_{s}^{\sigma}\right)^{\prime}\left(\mathbf{R}^{d}\right)$ is given by

$$
\left(V_{\phi} f\right)(x, \xi)=(2 \pi)^{-\frac{d}{2}}\left(f, \phi(\cdot-x) e^{i\langle\cdot, \xi\rangle}\right)_{L^{2}} .
$$

Here $(\cdot, \cdot)_{L^{2}}$ is the unique extension of the $L^{2}$-form on $\mathcal{S}_{s}^{\sigma}\left(\mathbf{R}^{d}\right)$ to a continuous sesqui-linear form on $\left(\mathcal{S}_{s}^{\sigma}\right)^{\prime}\left(\mathbf{R}^{d}\right) \times \mathcal{S}_{s}^{\sigma}\left(\mathbf{R}^{d}\right)$. In the case $f \in L^{p}\left(\mathbf{R}^{d}\right)$, for some $p \in[1, \infty]$, then $V_{\phi} f$ is given by

$$
V_{\phi} f(x, \xi) \equiv(2 \pi)^{-\frac{d}{2}} \int_{\mathbf{R}^{d}} f(y) \overline{\phi(y-x)} e^{-i\langle y, \xi\rangle} d y .
$$

The following characterizations of the $\mathcal{S}_{s_{1}, s_{2}}^{\sigma_{1}, \sigma_{2}}\left(\mathbf{R}^{d_{1}+d_{2}}\right), \Sigma_{s_{1}, s_{2}}^{\sigma_{1}, \sigma_{2}}\left(\mathbf{R}^{d_{1}+d_{2}}\right)$ and their duals follow by similar arguments as in the proofs of Propositions 2.1 and 2.2 in [41]. The details are left for the reader.

Proposition 1.4. Let $s_{j}, \sigma_{j}>0$ be such that $s_{j}+\sigma_{j} \geq 1, j=1,2$, $s_{0} \leq s$ and $\sigma_{0} \leq \sigma$. Also let $\phi \in \mathcal{S}_{s_{1}, s_{2}}^{\sigma_{1}, \sigma_{2}}\left(\mathbf{R}^{d_{1}+d_{2}} \backslash 0\right)\left(\phi \in \Sigma_{s_{1}, s_{2}}^{\sigma_{1}, \sigma_{2}}\left(\mathbf{R}^{d_{1}+d_{2}} \backslash\right.\right.$ 0 ) and let $f$ be a Gelfand-Shilov distribution on $\mathbf{R}^{d_{1}+d_{2}}$. Then $f \in$ $\mathcal{S}_{s_{1}, s_{2}}^{\sigma_{1}, \sigma_{2}}\left(\mathbf{R}^{d_{1}+d_{2}}\right)\left(f \in \Sigma_{s_{1}, s_{2}}^{\sigma_{1}, \sigma_{2}}\left(\mathbf{R}^{d_{1}+d_{2}}\right)\right)$, if and only if

$$
\left|V_{\phi} f\left(x_{1}, x_{2}, \xi_{1}, \xi_{2}\right)\right| \lesssim e^{-r\left(\left|x_{1}\right|^{\frac{1}{s_{1}}}+\left|x_{2}\right|^{\frac{1}{s_{2}}}+\left|\xi_{1}\right|^{\frac{1}{\sigma_{1}}}+\left|\xi_{2}\right|^{\frac{1}{\sigma_{2}}}\right)}
$$

holds for some $r>0$ (holds for every $r>0$ ).

A proof of Proposition 1.4 can be found in e. g. 24] (cf. 24, Theorem 2.7]). The corresponding result for Gelfand-Shilov distributions is the following improvement of [39, Theorem 2.5].

Proposition 1.5. Let $s_{j}, \sigma_{j}>0$ be such that $s_{j}+\sigma_{j} \geq 1, j=1,2$, $s_{0} \leq s$ and $t_{0} \leq t$. Also let $\phi \in \mathcal{S}_{s_{1}, s_{2}}^{\sigma_{1}, \sigma_{2}}\left(\mathbf{R}^{d_{1}+d_{2}}\right) \backslash 0$ and let $f$ be $a$ Gelfand-Shilov distribution on $\mathbf{R}^{d_{1}+d_{2}}$. Then the following is true:

(1) $f \in\left(\mathcal{S}_{s_{1}, s_{2}}^{\sigma_{1}, \sigma_{2}}\right)^{\prime}\left(\mathbf{R}^{d_{1}+d_{2}}\right)$, if and only if

$$
\left|V_{\phi} f\left(x_{1}, x_{2}, \xi_{1}, \xi_{2}\right)\right| \lesssim e^{r\left(\left|x_{1}\right|^{\frac{1}{s_{1}}}+\left|x_{2}\right|^{\frac{1}{s_{2}}}+\left|\xi_{1}\right|^{\frac{1}{\sigma_{1}}}+\left|\xi_{2}\right|^{\frac{1}{\sigma_{2}}}\right)}
$$

holds for every $r>0$;

(2) if in addition $\phi \in \Sigma_{s_{1}, s_{2}}^{\sigma_{1}, \sigma_{2}}\left(\mathbf{R}^{d_{1}+d_{2}}\right) \backslash 0$, then $f \in\left(\sum_{s_{1}, s_{2}}^{\sigma_{1}, \sigma_{2}}\right)^{\prime}\left(\mathbf{R}^{d_{1}+d_{2}}\right)$, if and only if

$$
\left|V_{\phi} f\left(x_{1}, x_{2}, \xi_{1}, \xi_{2}\right)\right| \lesssim e^{r\left(\left|x_{1}\right|^{\frac{1}{s_{1}}}+\left|x_{2}\right|^{\frac{1}{s_{2}}}+\left|\xi_{1}\right|^{\frac{1}{\sigma_{1}}}+\left|\xi_{2}\right|^{\frac{1}{\sigma_{2}}}\right)}
$$

holds for some $r>0$. 


\subsection{Broader family of modulation spaces.}

Definition 1.6. Let $\mathscr{B}$ be a Banach space of measurable functions on $\mathbf{R}^{d}$, and let $v \in \mathscr{P}_{E}\left(\mathbf{R}^{d}\right)$. Then $\mathscr{B}$ is called a translation invariant Banach Function space on $\mathbf{R}^{d}$ (with respect to $v$ ), or invariant $B F$ space on $\mathbf{R}^{d}$, if there is a constant $C$ such that the following conditions are fulfilled:

(1) if $x \in \mathbf{R}^{d}$ and $f \in \mathscr{B}$, then $f(\cdot-x) \in \mathscr{B}$, and

$$
\|f(\cdot-x)\|_{\mathscr{B}} \leq C v(x)\|f\|_{\mathscr{B}} ;
$$

(2) if $f, g \in L_{l o c}^{1}\left(\mathbf{R}^{d}\right)$ satisfy $g \in \mathscr{B}$ and $|f| \leq|g|$, then $f \in \mathscr{B}$ and

$$
\|f\|_{\mathscr{B}} \leq C\|g\|_{\mathscr{B}}
$$

(3) Minkowski's inequality holds true, i. e.

$$
\|f * \varphi\|_{\mathscr{B}} \lesssim\|f\|_{\mathscr{B}}\|\varphi\|_{L_{(v)}^{1}}, \quad f \in \mathscr{B}, \varphi \in L_{(v)}^{1}\left(\mathbf{R}^{d}\right) .
$$

If $v$ belongs to $\mathscr{P}_{E, s}\left(\mathbf{R}^{d}\right)\left(\mathscr{P}_{E, s}^{0}\left(\mathbf{R}^{d}\right)\right)$, then $\mathscr{B}$ in Definition [1.6] is called an invariant BF-space of Roumieu type (Beurling type) of order $s$.

It follows from (2) in Definition 1.6 that if $f \in \mathscr{B}$ and $h \in L^{\infty}$, then $f \cdot h \in \mathscr{B}$, and

$$
\|f \cdot h\|_{\mathscr{B}} \leq C\|f\|_{\mathscr{B}}\|h\|_{L^{\infty}} .
$$

In Definition 1.6, condition (2) means that a translation invariant BFspace is a solid BF-space in the sense of (A.3) in [12].

Example 1.7. Assume that $p, q \in[1, \infty]$, and let $L_{1}^{p, q}\left(\mathbf{R}^{2 d}\right)$ be the set of all $f \in L_{l o c}^{1}\left(\mathbf{R}^{2 d}\right)$ such that

$$
\|f\|_{L_{1}^{p, q}} \equiv\left(\int\left(\int|f(x, \xi)|^{p} d x\right)^{q / p} d \xi\right)^{1 / q}
$$

if finite. Then it follows that $L_{1}^{p, q}$ is translation invariant BF-spaces with respect to $v=1$.

We refer to [11, 15, 18, 22, 34, 40] for more facts about modulation spaces. Next we consider the extended class of modulation spaces which we are interested in.

Definition 1.8. Assume that $\mathscr{B}$ is a translation invariant QBF-space on $\mathbf{R}^{2 d}, \omega \in \mathscr{P}_{E}\left(\mathbf{R}^{2 d}\right)$, and that $\phi \in \Sigma_{1}\left(\mathbf{R}^{d}\right) \backslash 0$. Then the set $M(\omega, \mathscr{B})$ consists of all $f \in \Sigma_{1}^{\prime}\left(\mathbf{R}^{d}\right)$ such that

$$
\|f\|_{M(\omega, \mathscr{B})} \equiv\left\|V_{\phi} f \omega\right\|_{\mathscr{B}}
$$

is finite. 
Obviously, we have $M_{(\omega)}^{p, q}\left(\mathbf{R}^{d}\right)=M(\omega, \mathscr{B})$ when $\mathscr{B}=L_{1}^{p, q}\left(\mathbf{R}^{2 d}\right)$ (cf. Example 1.7). It follows that many properties which are valid for the classical modulation spaces also hold for the spaces of the form $M(\omega, \mathscr{B})$.

We notice that $M(\omega, \mathscr{B})$ is independent of the choice of $\phi$ in Definition 1.8 cf. [43]. Furthermore, $M(\omega, \mathscr{B})$ is a Banach space in view of $[28$.

1.5. Pseudo-differential operators. Next we recall some facts on pseudo-differential operators. Let $A \in \mathbf{M}(d, \mathbf{R})$ be fixed and let $a \in$ $\Sigma_{1}\left(\mathbf{R}^{2 d}\right)$. Then the pseudo-differential operator $\operatorname{Op}_{A}(a)$ is the linear and continuous operator on $\Sigma_{1}\left(\mathbf{R}^{d}\right)$, defined by the formula

$$
\begin{aligned}
& \left(\mathrm{Op}_{A}(a) f\right)(x) \\
& \quad=(2 \pi)^{-d} \iint a(x-A(x-y), \xi) f(y) e^{i\langle x-y, \xi\rangle} d y d \xi
\end{aligned}
$$

The definition of $\mathrm{Op}_{A}(a)$ extends to any $a \in \Sigma_{1}^{\prime}\left(\mathbf{R}^{2 d}\right)$, and then $\mathrm{Op}_{A}(a)$ is continuous from $\Sigma_{1}\left(\mathbf{R}^{d}\right)$ to $\Sigma_{1}^{\prime}\left(\mathbf{R}^{d}\right)$. Moreover, for every fixed $A \in$ $\mathbf{M}(d, \mathbf{R})$, it follows that there is a one to one correspondence between such operators and pseudo-differential operators of the form $\mathrm{Op}_{A}(a)$. (See e.g. [26].) If $A=2^{-1} I$, where $I \in \mathbf{M}(d, \mathbf{R})$ is the identity matrix, then $\mathrm{Op}_{A}(a)$ is equal to the Weyl operator $\mathrm{Op}^{w}(a)$ of $a$. If instead $A=0$, then the standard (Kohn-Nirenberg) representation $\operatorname{Op}(a)$ is obtained.

If $a_{1}, a_{2} \in \Sigma_{1}^{\prime}\left(\mathbf{R}^{2 d}\right)$ and $A_{1}, A_{2} \in \mathbf{M}(d, \mathbf{R})$, then

$$
\mathrm{Op}_{A_{1}}\left(a_{1}\right)=\mathrm{Op}_{A_{2}}\left(a_{2}\right) \quad \Leftrightarrow \quad a_{2}(x, \xi)=e^{i\left\langle\left(A_{1}-A_{2}\right) D_{\xi}, D_{x}\right\rangle} a(x, \xi) .
$$

(Cf. [26].)

1.6. Symbol classes. Next we introduce function spaces related to symbol classes of the pseudo-differential operators. These functions should obey various conditions of the form

$$
\left|\partial_{x}^{\alpha} \partial_{\xi}^{\beta} a(x, \xi)\right| \lesssim h^{|\alpha+\beta|} \alpha !^{\sigma} \beta !^{s} \omega(x, \xi),
$$

for functions on $\mathbf{R}^{d_{1}+d_{2}}$. For this reason we consider semi-norms of the form

$$
\|a\|_{\Gamma_{(\omega)}^{\sigma, s ; h}} \equiv \sup _{(\alpha, \beta) \in \mathbf{N}^{d_{1}+d_{2}}}\left(\sup _{(x, \xi) \in \mathbf{R}^{d_{1}+d_{2}}}\left(\frac{\left|\partial_{x}^{\alpha} \partial_{\xi}^{\beta} a(x, \xi)\right|}{h^{|\alpha+\beta|} \alpha !^{\sigma} \beta !^{s} \omega(x, \xi)}\right)\right)
$$

indexed by $h>0$,

Definition 1.9. Let $s, \sigma$ and $h$ be positive constants, let $\omega$ be a weight on $\mathbf{R}^{d_{1}+d_{2}}$, and let

$$
\omega_{r}(x, \xi) \equiv e^{r\left(|x|^{\frac{1}{s}}+|\xi|^{\frac{1}{\sigma}}\right)}
$$


(1) The set $\Gamma_{(\omega)}^{\sigma, s ; h}\left(\mathbf{R}^{d_{1}+d_{2}}\right)$ consists of all $a \in C^{\infty}\left(\mathbf{R}^{d_{1}+d_{2}}\right)$ such that $\|a\|_{\Gamma_{(\omega)}^{\sigma, s ; h}}$ in (1.15) is finite. The set $\Gamma_{0}^{\sigma, s ; h}\left(\mathbf{R}^{d_{1}+d_{2}}\right)$ consists of all $a \in C^{\infty}\left(\mathbf{R}^{d_{1}+d_{2}}\right)$ such that $\|a\|_{\Gamma_{\left(\omega_{r}\right)}^{\sigma, s ; h}}$ is finite for every $r>0$, and the topology is the projective limit topology of $\Gamma_{\left(\omega_{r}\right)}^{\sigma, s ; h}\left(\mathbf{R}^{d_{1}+d_{2}}\right)$ with respect to $r>0$;

(2) The sets $\Gamma_{(\omega)}^{\sigma, s}\left(\mathbf{R}^{d_{1}+d_{2}}\right)$ and $\Gamma_{(\omega)}^{\sigma, s ; 0}\left(\mathbf{R}^{d_{1}+d_{2}}\right)$ are given by

$$
\Gamma_{(\omega)}^{\sigma, s}\left(\mathbf{R}^{d_{1}+d_{2}}\right) \equiv \bigcup_{h>0} \Gamma_{(\omega)}^{\sigma, s ; h}\left(\mathbf{R}^{d_{1}+d_{2}}\right)
$$

and

$$
\Gamma_{(\omega)}^{\sigma, s ; 0}\left(\mathbf{R}^{d_{1}+d_{2}}\right) \equiv \bigcap_{h>0} \Gamma_{(\omega)}^{\sigma, s ; h}\left(\mathbf{R}^{d_{1}+d_{2}}\right)
$$

and their topologies are the inductive respective the projective topologies of $\Gamma_{(\omega)}^{\sigma, s ; h}\left(\mathbf{R}^{d_{1}+d_{2}}\right)$ with respect to $h>0$.

The following result is a straight-forward consequence of [1, Proposition 2.4] and the definitions.

Proposition 1.10. Let $R>0, q \in(0, \infty], s, \sigma>0$ be such that $s+\sigma \geq 1$ and $(s, \sigma) \neq\left(\frac{1}{2}, \frac{1}{2}\right), \phi \in \Sigma_{s, \sigma}^{\sigma, s}\left(\mathbf{R}^{2 d}\right) \backslash 0, \omega \in \mathscr{P}_{s, \sigma}\left(\mathbf{R}^{2 d}\right)$, and let

$$
\omega_{R}(x, \xi, \eta, y)=\omega(x, \xi) e^{-R\left(|y|^{\frac{1}{s}}+|\eta|^{\frac{1}{\sigma}}\right)} .
$$

Then

$$
\begin{aligned}
\Gamma_{(\omega)}^{\sigma, s}\left(\mathbf{R}^{2 d}\right) & =\bigcup_{R>0}\left\{a \in\left(\Sigma_{s, \sigma}^{\sigma, s}\right)^{\prime}\left(\mathbf{R}^{2 d}\right) ;\left\|\omega_{R}^{-1} V_{\phi} a\right\|_{L^{\infty, q}}<\infty\right\}, \\
\Gamma_{(\omega)}^{\sigma, s ; 0}\left(\mathbf{R}^{2 d}\right) & =\bigcap_{R>0}\left\{a \in\left(\Sigma_{s, \sigma}^{\sigma, s}\right)^{\prime}\left(\mathbf{R}^{2 d}\right) ;\left\|\omega_{R}^{-1} V_{\phi} a\right\|_{L^{\infty, q}}<\infty\right\} .
\end{aligned}
$$

The following lemma is a consequence of [1, Theorem 3.6].

Lemma 1.11. Let $s, \sigma>0$ such that $s+\sigma \geq 1 \omega \in \mathscr{P}_{s, \sigma}\left(\mathbf{R}^{2 d}\right), A_{1}, A_{2} \in$ $\mathbf{M}(d, \mathbf{R})$, and that $a_{1}, a_{2} \in\left(\Sigma_{s, \sigma}^{\sigma, s}\right)^{\prime}\left(\mathbf{R}^{2 d}\right)$ are such that $\mathrm{Op}_{A_{1}}\left(a_{1}\right)=$ $\mathrm{Op}_{A_{2}}\left(a_{2}\right)$. Then

$$
a_{1} \in \Gamma_{(\omega)}^{\sigma, s ; 0}\left(\mathbf{R}^{2 d}\right) \quad \Leftrightarrow \quad a_{2} \in \Gamma_{(\omega)}^{\sigma, s ; 0}\left(\mathbf{R}^{2 d}\right)
$$

and similarly for $\Gamma_{(\omega)}^{\sigma, s}\left(\mathbf{R}^{2 d}\right)$ in place of $\Gamma_{(\omega)}^{\sigma, s ; 0}\left(\mathbf{R}^{2 d}\right)$.

2. Continuity for pseudo-Differential operators With SYMBOLS OF INFINITE ORDER

In this section we discuss continuity for operators in $\operatorname{Op}\left(\Gamma_{\left(\omega_{0}\right)}^{\sigma, s}\right)$ and $\operatorname{Op}\left(\Gamma_{\left(\omega_{0}\right)}^{\sigma, s ; 0}\right)$ when acting on a general class of modulation spaces. In Theorem 2.1 continuity is treated where the symbols belong to $\Gamma_{\left(\omega_{0}\right)}^{\sigma, s}$ and in 
Theorem 2.5 continuity is treated where the symbols belong to $\Gamma_{\left(\omega_{0}\right)}^{\sigma, s ; 0}$. This gives an analogy to [38, Theorem 3.2] in the framework of operator theory and Gelfand-Shilov classes.

Our main result is stated as follows.

Theorem 2.1. Let $A \in \mathbf{M}(d, \mathbf{R}), s, \sigma \geq 1, \omega, \omega_{0} \in \mathscr{P}_{s, \sigma}^{0}\left(\mathbf{R}^{2 d}\right), a \in$ $\Gamma_{\left(\omega_{0}\right)}^{\sigma, s}\left(\mathbf{R}^{2 d}\right)$, and that $\mathscr{B}$ is an invariant BF-space on $\mathbf{R}^{2 d}$. Then $\mathrm{Op}_{A}(a)$ is continuous from $M\left(\omega_{0} \omega, \mathscr{B}\right)$ to $M(\omega, \mathscr{B})$.

We need some preparations for the proof, and start with the following remark.

Remark 2.2. Let $s, \sigma>0$ such that $s+\sigma \geq 1$. If $a \in\left(\Sigma_{s, \sigma}^{\sigma, s}\right)^{\prime}\left(\mathbf{R}^{2 d}\right)$, then there is a unique $b \in\left(\sum_{s, \sigma}^{\sigma, s}\right)^{\prime}\left(\mathbf{R}^{2 d}\right)$ such that $\mathrm{Op}(a)^{*}=\mathrm{Op}(b)$, where $b(x, \xi)=e^{i\left\langle D_{\xi}, D_{x}\right\rangle} \overline{a(x, \xi)}$ in view of [26, Theorem 18.1.7]. Furthermore, by the latter equality and [3, Theorem 4.1] it follows that

$$
a \in \Gamma_{(\omega)}^{\sigma, s}\left(\mathbf{R}^{2 d}\right) \quad \Leftrightarrow \quad b \in \Gamma_{(\omega)}^{\sigma, s}\left(\mathbf{R}^{2 d}\right) .
$$

Lemma 2.3. Suppose $s, \sigma \geq 1, \omega \in \mathscr{P}_{E}\left(\mathbf{R}^{d_{0}}\right)$ and that $f \in C^{\infty}\left(\mathbf{R}^{d+d_{0}}\right)$ satisfies

$$
\left|\partial^{\alpha} f(x, y)\right| \lesssim h^{|\alpha|} \alpha !^{\sigma} e^{-r|x|^{\frac{1}{s}}} \omega(y), \alpha \in \mathbf{N}^{d+d_{0}}
$$

for some $h>0$ and $r>0$. Then there are $f_{0} \in C^{\infty}\left(\mathbf{R}^{d+d_{0}}\right)$ and $\psi \in \mathcal{S}_{s}^{\sigma}\left(\mathbf{R}^{d}\right)$ such that (2.1) holds with $f_{0}$ in place of $f$ for some for some $h>0$ and $r>0$, and $f(x, y)=f_{0}(x, y) \psi(x)$.

Proof. By Proposition [1.1, there is a submultiplicative weight $v_{0} \in$ $\mathscr{P}_{E, s}\left(\mathbf{R}^{d}\right) \cap C^{\infty}\left(\mathbf{R}^{d}\right)$ such that

$$
v_{0}(x) \asymp e^{\frac{r}{2}|x|^{\frac{1}{s}}}
$$

and

$$
\left|\partial^{\alpha} v_{0}(x)\right| \lesssim h^{|\alpha|} \alpha !^{\sigma} v_{0}(x), \quad \alpha \in \mathbf{N}^{d}
$$

for some $h, r>0$. Since $s, \sigma \geq 1$, a straight-forward application of Faà di Bruno's formula, for the composed function $\psi(x)=g\left(v_{0}(x)\right)$, where $g(t)=\frac{1}{t}$, on (2.3) gives

$$
\left|\partial^{\alpha}\left(\frac{1}{v_{0}(x)}\right)\right| \lesssim h^{|\alpha|} \alpha !^{\sigma} \cdot \frac{1}{v_{0}(x)}, \quad \alpha \in \mathbf{N}^{d}
$$

for some $h>0$. It follows from (2.2) and (2.3) $)^{\prime}$ that if $\psi=1 / v_{0}$, then $\psi \in \mathcal{S}_{s}^{\sigma}\left(\mathbf{R}^{d}\right)$. Furthermore, if $f_{0}(x, y)=f(x, y) v_{0}(x)$, then an 
application of Leibnitz formula we get

$$
\begin{aligned}
&\left|\partial_{x}^{\alpha} \partial_{y}^{\alpha_{0}} f_{0}(x, y)\right| \lesssim \sum_{\gamma \leq \alpha}\left(\begin{array}{l}
\alpha \\
\gamma
\end{array}\right)\left|\partial_{x}^{\delta} \partial_{y}^{\alpha_{0}} f(x, y)\right|\left|\partial^{\alpha-\delta} v_{0}(x)\right| \\
& \lesssim h^{|\alpha|+\left|\alpha_{0}\right|} \sum_{\gamma \leq \alpha}\left(\begin{array}{l}
\alpha \\
\gamma
\end{array}\right)\left(\gamma ! \alpha_{0} !\right)^{\sigma} e^{-r|x|^{\frac{1}{s}}} \omega(y)(\alpha-\gamma) !^{\sigma} v_{0}(x) \\
& \lesssim(2 h)^{|\alpha|+\left|\alpha_{0}\right|}\left(\alpha ! \alpha_{0} !\right)^{\sigma} e^{-r|x|^{\frac{1}{s}}} v_{0}(x) \omega(y) \\
& \lesssim(2 h)^{|\alpha|+\left|\alpha_{0}\right|}\left(\alpha ! \alpha_{0} !\right)^{\sigma} e^{-\frac{r}{2}|x|^{\frac{1}{s}}} \omega(y)
\end{aligned}
$$

for some $h>0$, which gives the desired estimate on $f_{0}$, since it is clear that $f(x, y)=f_{0}(x, y) \psi(x)$.

Lemma 2.4. Let $s, \sigma \geq 1, \omega \in \mathscr{P}_{s, \sigma}^{0}\left(\mathbf{R}^{2 d}\right), v_{1} \in \mathscr{P}_{s}^{0}\left(\mathbf{R}^{d}\right)$ and $v_{2} \in$ $\mathscr{P}_{\sigma}^{0}\left(\mathbf{R}^{d}\right)$ be such that $v_{1}$ and $v_{2}$ are submultiplicative, $\omega \in \Gamma_{(\omega)}^{\sigma, s}\left(\mathbf{R}^{2 d}\right)$ is $v_{1} \otimes v_{2}$-moderate. Also let $a \in \Gamma_{(\omega)}^{\sigma, s}\left(\mathbf{R}^{2 d}\right), f \in \mathcal{S}_{s}^{\sigma}\left(\mathbf{R}^{d}\right), \phi \in \Sigma_{s}^{\sigma}\left(\mathbf{R}^{d}\right)$, $\phi_{2}=\phi v_{1}$, If

$$
\Phi(x, \xi, z, \zeta)=\frac{a(x+z, \xi+\zeta)}{\omega(x, \xi) v_{1}(z) v_{2}(\zeta)}
$$

and

$$
H(x, \xi, y)=\iint \Phi(x, \xi, z, \zeta) \phi_{2}(z) v_{2}(\zeta) e^{i\langle y-x-z, \zeta\rangle} d z d \zeta
$$

Then

$$
V_{\phi}(\mathrm{Op}(a) f)(x, \xi)=(2 \pi)^{-d}\left(f, e^{i\langle\cdot, \xi\rangle} H(x, \xi, \cdot)\right) \omega(x, \xi) .
$$

Furthermore the following is true:

(1) $H \in C^{\infty}\left(\mathbf{R}^{3 d}\right)$ and satisfies

$$
\left|\partial_{y}^{\alpha} H(x, \xi, y)\right| \lesssim h_{0}^{|\alpha|} \alpha !^{\sigma} e^{-r_{0}|x-y|^{\frac{1}{s}}},
$$

for every $\alpha \in \mathbf{R}^{d}$ and some $h_{0}, r_{0}>0$;

(2) there are functions $H_{0} \in C^{\infty}\left(\mathbf{R}^{3 d}\right)$ and $\phi_{0} \in \mathcal{S}_{s}^{\sigma}\left(\mathbf{R}^{d}\right)$ such that

$$
H(x, \xi, y)=H_{0}(x, \xi, y) \phi_{0}(y-x),
$$

and such that (2.7) holds for some $h_{0}, r_{0}>0$, with $H_{0}$ in place of $H$.

Lemma 2.4 follows by similar arguments as in [43]. In order to be self contained we give a different proof.

Proof. By straight-forward computations we get

$$
V_{\phi}(\mathrm{Op}(a) f)(x, \xi)=(2 \pi)^{-d}\left(f, e^{i\langle\cdot, \xi\rangle} H_{1}(x, \xi, \cdot)\right) \omega(x, \xi),
$$


where

$$
\begin{gathered}
H_{1}(x, \xi, y)=(2 \pi)^{d} e^{-i\langle y, \xi\rangle}\left(\operatorname{Op}(a)^{*}\left(\phi(\cdot-x) e^{i\langle\cdot \xi\rangle}\right)\right)(y) / \omega(x, \xi) \\
=\iint \frac{a(z, \zeta)}{\omega(x, \xi)} \phi(z-x) e^{i\langle y-z, \zeta-\xi\rangle} d z d \zeta \\
=\iint \Phi(x, \xi, z-x, \zeta-\xi) \phi_{2}(z-x) v_{2}(\zeta-\xi) e^{i\langle y-z, \zeta-\xi\rangle} d z d \zeta .
\end{gathered}
$$

If $z-x$ and $\zeta-\xi$ are taken as new variables of integrations, it follows that the right-hand side is the same as (2.5). Hence (2.6) holds. This gives the first part of the lemma.

The smoothness of $H$ is a consequence of the uniqueness of the adjoint (cf. Remark 2.2) and [43, Lemma 2.7].

To show that (2.7) holds, let

$$
\Phi_{0}(x, \xi, z, \zeta)=\Phi(x, \xi, z, \zeta) \phi_{2}(z)
$$

where $\Phi$ defined as in (2.4), and let $\Psi=\mathscr{F}_{3} \Phi_{0}$, where $\mathscr{F}_{3} \Phi_{0}$ is the partial Fourier transform of $\Phi_{0}(x, \xi, z, \zeta)$ with respect to the $z$ variable. Then it follows from the assumptions and $(2.3)^{\prime}$ that

$$
\begin{aligned}
&\left|\partial_{z}^{\alpha} \Phi_{0}(x, \xi, z, \zeta)\right| \lesssim \sum_{\gamma \leq \alpha}\left(\begin{array}{l}
\alpha \\
\gamma
\end{array}\right) \sum_{\lambda \leq \gamma}\left(\begin{array}{l}
\gamma \\
\lambda
\end{array}\right) \frac{\left|\partial_{z}^{\gamma-\lambda} a(x+z, \xi+\zeta)\right|}{\omega(x, \xi) v_{2}(\zeta)} \\
& \times \partial^{\lambda}\left(\frac{1}{v_{1}(z)}\right) h^{|\alpha-\gamma|}(\alpha-\gamma) !^{\sigma} e^{-r|z|^{\frac{1}{s}}} \\
& \lesssim \sum_{\gamma \leq \alpha}\left(\begin{array}{l}
\alpha \\
\gamma
\end{array}\right) \sum_{\lambda \leq \gamma}\left(\begin{array}{l}
\gamma \\
\lambda
\end{array}\right) h^{|\alpha|}(\alpha-\gamma) !^{\sigma}(\gamma-\lambda) !^{\sigma} \lambda !^{\sigma} e^{-r_{0}|z|^{\frac{1}{s}}} \\
& \lesssim h^{|\alpha|} \alpha !^{\sigma} \sum_{\gamma \leq \alpha}\left(\begin{array}{l}
\alpha \\
\gamma
\end{array}\right) \sum_{\lambda \leq \gamma}\left(\begin{array}{l}
\gamma \\
\lambda
\end{array}\right)\left(\frac{(\alpha-\gamma) ! \gamma !}{\alpha !}\right)^{\sigma}\left(\frac{(\gamma-\lambda) ! \lambda !}{\gamma !}\right)^{\sigma} e^{-r_{0}|z|^{\frac{1}{s}}} \\
& \lesssim(4 h)^{|\alpha|} \alpha !^{\sigma} e^{-r|z|^{\frac{1}{s}}} \sum_{\gamma \leq \alpha} 1 \cdot \sum_{\lambda \leq \gamma} 1 .
\end{aligned}
$$

Since $\sum_{\lambda \leq \gamma} 1 \lesssim 2^{|\gamma|}$, we get

$$
\left|\partial_{z}^{\alpha} \Phi_{0}(x, \xi, z, \zeta)\right| \leq C(16 h)^{|\alpha|} \alpha !^{\sigma} e^{-r_{0}|z|^{\frac{1}{s}}} \leq C h_{0}^{|\alpha|} \alpha !^{\sigma} e^{-r_{0}|z|^{\frac{1}{s}}}
$$

for some $C, h_{0}, r_{0}>0$. Then $z \mapsto \Phi_{0}(x, \xi, z, \zeta)$ is an element in $\mathcal{S}_{s}^{\sigma}\left(\mathbf{R}^{d}\right)$. Moreover, $\left\{\Phi_{0}(x, \xi, z, \zeta)\right\}_{z \in \mathbf{R}^{d}}$ is a bounded set in $\Gamma_{(1)}^{\sigma, s}\left(\mathbf{R}^{d} \times \mathbf{R}^{2 d}\right)$. Indeed, for a fixed $z_{0} \in \mathbf{R}^{d}$, then an application of Leibnitz formula, Faà 
di Bruno's formula, Proposition 1.1 and $(2.3)^{\prime}$, give

$$
\begin{aligned}
& \left|\partial_{x}^{\alpha} \partial_{\xi}^{\beta} \partial_{\zeta}^{\gamma} \Phi_{0}\left(x, \xi, z_{0}, \zeta\right)\right| \leq \sum\left(\begin{array}{c}
\alpha \\
\alpha_{1}
\end{array}\right)\left(\begin{array}{c}
\beta \\
\beta_{1}
\end{array}\right)\left(\begin{array}{c}
\gamma \\
\gamma_{1}
\end{array}\right) \partial_{x}^{\alpha_{1}} \partial_{\xi}^{\beta_{1}}\left(\frac{1}{\omega(x, \xi)}\right) \\
& \times \partial_{\zeta}^{\gamma_{1}}\left(\frac{1}{v_{2}(\zeta)}\right)\left|\partial_{x}^{\alpha-\alpha_{1}} \partial_{\xi}^{\beta-\beta_{1}} \partial_{\zeta}^{\gamma-\gamma_{1}} a\left(x+z_{0}, \xi+\zeta\right)\right| \cdot \frac{\left|\phi\left(z_{0}\right)\right|}{v_{1}\left(z_{0}\right)} \\
& \lesssim \sum\left(\begin{array}{c}
\alpha \\
\alpha_{1}
\end{array}\right)\left(\begin{array}{c}
\beta \\
\beta_{1}
\end{array}\right)\left(\begin{array}{c}
\gamma \\
\gamma_{1}
\end{array}\right) h^{\left|\alpha_{1}+\beta_{1}+\gamma_{1}\right|} \alpha_{1} !^{\sigma}\left(\beta_{1} ! \gamma_{1} !\right)^{s} \\
& \times\left(\frac{1}{\omega(x, \xi) v_{1}\left(z_{0}\right) v_{2}(\zeta)}\right)\left|\partial_{x}^{\alpha-\alpha_{1}} \partial_{\xi}^{\beta-\beta_{1}} \partial_{\zeta}^{\gamma-\gamma_{1}} a\left(x+z_{0}, \xi+\zeta\right)\right| \\
& \lesssim h^{|\alpha+\beta+\gamma|} \sum\left(\begin{array}{c}
\alpha \\
\alpha_{1}
\end{array}\right)\left(\begin{array}{c}
\beta \\
\beta_{1}
\end{array}\right)\left(\begin{array}{c}
\gamma \\
\gamma_{1}
\end{array}\right)\left(\left(\alpha-\alpha_{1}\right) ! \alpha_{1} !\right)^{\sigma}\left(\left(\beta-\beta_{1}\right) ! \beta_{1} !\right)^{s}\left(\left(\gamma-\gamma_{1}\right) ! \gamma_{1} !\right)^{s} \\
& \lesssim(4 h)^{|\alpha+\beta+\gamma|} \alpha !^{\sigma}(\beta ! \gamma !)^{s},
\end{aligned}
$$

where all the summations above are taken over all $\alpha_{1} \leq \alpha, \beta_{1} \leq \beta$ and $\gamma_{1} \leq \gamma$. In view of Proposition 1.2 and (2.10) we have

$$
\left|\partial_{\eta}^{\alpha} \Psi(x, \xi, \eta, \zeta)\right| \lesssim h_{0}^{|\alpha|} \alpha !^{s} e^{-r_{0}|\eta|^{\frac{1}{\sigma}}}
$$

for some $h_{0}, r_{0}>0$. Hence

$$
\left|\partial_{\eta}^{\alpha}\left(\Psi(x, \xi, \zeta, \zeta) v_{2}(\zeta)\right)\right| \lesssim h_{0}^{|\alpha|} \alpha !^{s} e^{-r_{0}|\zeta|^{\frac{1}{\sigma}}}
$$

for some $h_{0}, r_{0}>0$.

By letting $H_{2}(x, \xi, \cdot)$ be the inverse partial Fourier transform of $\Psi(x, \xi, \zeta, \zeta) v_{2}(\zeta)$ with respect to the $\zeta$ variable, it follows that

$$
\left|\partial_{y}^{\alpha} H_{2}(x, \xi, y)\right| \lesssim h_{0}^{|\alpha|} \alpha !^{\sigma} e^{-r_{0}|y|^{\frac{1}{s}}}
$$

for some $h_{0}, r_{0}>0$. The assertion (1) now follows from the latter estimate and the fact that $H(x, \xi, y)=H_{2}(x, \xi, x-y)$.

In order to prove (2) we notice that (2.11) shows that $y \mapsto H_{2}(x, \xi, y)$ is an element in $\mathcal{S}_{s}^{\sigma}\left(\mathbf{R}^{d}\right)$ with values in $\Gamma_{s, \sigma}^{(1)}\left(\mathbf{R}^{2 d}\right)$. It follows by Lemma 2.3 that there exist $H_{3} \in C^{\infty}\left(\mathbf{R}^{3 d}\right)$ and $\phi_{0} \in \mathcal{S}_{s}^{\sigma}\left(\mathbf{R}^{d}\right)$ such that (2.11) holds for some $h_{0}, r_{0}>0$ with $H_{3}$ in place of $H_{2}$, and

$$
H_{2}(x, \xi, y)=H_{3}(x, \xi, y) \phi_{0}(-y) \text {. }
$$

This is the same as (2), and the result follows.

Proof of Theorem [2.1. There is no restriction if we assume that $A=0$. Let $G=\operatorname{Op}(a) f$. In view of Lemma 2.4 we have

$$
\begin{aligned}
V_{\phi} G(x, \xi)=(2 \pi)^{-\frac{d}{2}} \mathscr{F}\left(\left(f \cdot \overline{\phi_{0}(\cdot-x)}\right) \cdot H_{0}(x, \xi, \cdot)\right)(\xi) \omega(x, \xi) \\
=(2 \pi)^{-d}\left(V_{\phi_{0}} f\right)(x, \cdot) *\left(\mathscr{F}\left(H_{0}(x, \xi, \cdot)\right)\right)(\xi) \omega(x, \xi) .
\end{aligned}
$$


Since $\omega$ and $\omega_{0}$ belong to $\mathscr{P}_{s, \sigma}^{0}\left(\mathbf{R}^{2 d}\right)$, then for every $r_{0}>0$ and $x, \xi, \eta \in$ $\mathbf{R}^{d}$ we have

$$
\omega(x, \xi) \omega_{0}(x, \xi) \lesssim \omega(x, \eta) \omega_{0}(x, \eta) e^{\frac{r_{0}}{2}|\xi-\eta|^{\frac{1}{\sigma}}},
$$

this inequality and (2) in Lemma 2.4 give

$$
\left|V_{\phi} G(x, \xi) \omega_{0}(x, \xi)\right| \lesssim\left(\left|\left(V_{\phi_{0}} f\right)(x, \cdot) \omega(x, \cdot) \omega_{0}(x, \cdot)\right| * e^{-\frac{r_{0}}{2}|\cdot| \frac{1}{\sigma}}\right)(\xi) .
$$

In view of Definition 1.6, we get for some $v \in \mathscr{P}_{\sigma}^{0}\left(\mathbf{R}^{d}\right)$,

$$
\begin{aligned}
\|G\|_{M\left(\omega_{0}, \mathscr{B}\right)} \lesssim\left\|\left|\left(V_{\phi_{0}} f\right) \cdot \omega \cdot \omega_{0}\right| * \delta_{0} \otimes e^{-r_{0}|\cdot| \frac{1}{\sigma}}\right\|_{\mathscr{B}} \\
\quad \leq\left\|\left(V_{\phi_{0}} f\right) \cdot \omega \cdot \omega_{0}\right\|_{\mathscr{B}}\left\|e^{-r_{0}|\cdot| \frac{1}{\sigma}} v\right\|_{L^{1}} \asymp\|f\|_{M\left(\omega \cdot \omega_{0}, \mathscr{B}\right)} .
\end{aligned}
$$

This gives the result.

By similar arguments as in the proof of Theorem 2.1 and Lemma 2.4 we get the following. The details are left for the reader.

Theorem 2.5. Let $A \in \mathbf{M}(d, \mathbf{R}), s, \sigma \geq 1, \omega, \omega_{0} \in \mathscr{P}_{s, \sigma}\left(\mathbf{R}^{2 d}\right)$, $a \in$ $\Gamma_{\left(\omega_{0}\right)}^{\sigma, s ; 0}\left(\mathbf{R}^{2 d}\right)$, and that $\mathscr{B}$ is an invariant BF-space on $\mathbf{R}^{2 d}$. Then $\mathrm{Op}_{A}(a)$ is continuous from $M\left(\omega_{0} \omega, \mathscr{B}\right)$ to $M(\omega, \mathscr{B})$.

Lemma 2.6. Let $s, \sigma \geq 1, \omega \in \mathscr{P}_{s, \sigma}\left(\mathbf{R}^{2 d}\right), v_{1} \in \mathscr{P}_{s}\left(\mathbf{R}^{d}\right)$ and $v_{2} \in$ $\mathscr{P}_{\sigma}\left(\mathbf{R}^{d}\right)$ be such that $v_{1}$ and $v_{2}$ are submultiplicative, $\omega \in \Gamma_{(\omega)}^{\sigma, s ; 0}\left(\mathbf{R}^{2 d}\right)$ is $v_{1} \otimes v_{2}$-moderate. Also let $a \in \Gamma_{(\omega)}^{\sigma, s ; 0}\left(\mathbf{R}^{2 d}\right), f, \phi \in \Sigma_{s}^{\sigma}\left(\mathbf{R}^{d}\right), \phi_{2}=\phi v_{1}$, and let $\Phi$ and $H$ be as in Lemma 2.4. Then (2.6) and the following hold true:

(1) $H \in C^{\infty}\left(\mathbf{R}^{3 d}\right)$ and satisfies (2.7) for every $h_{0}, r_{0}>0$;

(2) there are functions $H_{0} \in C^{\infty}\left(\mathbf{R}^{3 d}\right)$ and $\phi_{0} \in \Sigma_{s}\left(\mathbf{R}^{d}\right)$ such that (2.8) holds, and such that (2.7) holds for every $h_{0}, r_{0}>0$, with $H_{0}$ in place of $H$.

\section{REFERENCES}

[1] A. Abdeljawad, M. Cappiello, J. Toft Pseudo-differential calculus in anisotropic Gelfand-Shilov setting, (preprint) arXiv:1805.03497 (2018).

[2] A. Abdeljawad, S. Coriasco, J. Toft Liftings for ultra-modulation spaces, and one-parameter groups of Gevrey type pseudo-differential operators, (preprint) arXiv:1712.04338 (2017).

[3] M. Cappiello, J. Toft Pseudo-differential operators in a Gelfand-Shilov setting, Math. Nachr. 290 (2017), 738-755.

[4] F. Concetti, J. Toft. Trace ideals for Fourier integral operators with nonsmooth symbols, "Pseudo-Differential Operators: Partial Differential Equations and Time-Frequency Analysis", Fields Inst. Commun., Amer. Math. Soc., 52 2007, pp.255-264.

[5] F. Concetti, G. Garello, J. Toft. Trace ideals for Fourier integral operators with non-smooth symbols II. Osaka J. Math., 47 (2010), 739-786.

[6] E. Cordero, K. Gröchenig, F. Nicola, L. Rodino. Wiener algebras of Fourier integral operators, J. Math. Pures Appl., (2013), 219-233. 
[7] E. Cordero, K. Gröchenig, F. Nicola and L. Rodino, Generalized Metaplectic Operators and the Schrödinger Equation with a Potential in the Sjöstrand Class, J. Math. Phys., 55, (2014), 081506:1-17.

[8] J. Chung, S.-Y. Chung, D. Kim, Characterizations of the Gelfand-Shilov spaces via Fourier transforms, Proc. Amer. Math. Soc. 124 (1996), 21012108.

[9] H. G. Feichtinger Banach spaces of distributions of Wiener's type and interpolation, in: Ed. P. Butzer, B. Sz. Nagy and E. Görlich (Eds), Proc. Conf. Oberwolfach, Functional Analysis and Approximation, August 1980, Int. Ser. Num. Math. 69 Birkhäuser Verlag, Basel, Boston, Stuttgart, 1981, pp. $153-165$.

[10] H. G. Feichtinger Banach convolution algebras of Wiener's type, in: Proc. Functions, Series, Operators in Budapest, Colloquia Math. Soc. J. Bolyai, North Holland Publ. Co., Amsterdam Oxford NewYork, 1980.

[11] H. G. Feichtinger Modulation spaces on locally compact abelian groups. Technical report, University of Vienna, Vienna, 1983; also in: M. Krishna, R. Radha, S. Thangavelu (Eds) Wavelets and their applications, Allied Publishers Private Limited, NewDehli Mumbai Kolkata Chennai Hagpur Ahmedabad Bangalore Hyderbad Lucknow, 2003, pp.99-140.

[12] H. G. Feichtinger Wiener amalgams over Euclidean spaces and some of their applications, in: Function spaces (Edwardsville, IL, 1990), Lect. Notes in pure and appl. math., 136, Marcel Dekker, New York, 1992, pp. 123-137.

[13] H. G. Feichtinger Modulation spaces: Looking back and ahead, Sampl. Theory Signal Image Process. 5 (2006), 109-140.

[14] H. G. Feichtinger Choosing function spaces in harmonic analysis, Excursions in harmonic analysis 4, Appl. Numer. Harmon. Anal., 65101, Birkhäuser/Springer, Cham, 2015.

[15] H. G. Feichtinger and K. H. Gröchenig Banach spaces related to integrable group representations and their atomic decompositions, I, J. Funct. Anal. 86 (1989), 307-340.

[16] H. G. Feichtinger and K. H. Gröchenig Banach spaces related to integrable group representations and their atomic decompositions, II, Monatsh. Math. 108 (1989), 129-148.

[17] H. G. Feichtinger and K. H. Gröchenig Gabor frames and time-frequency analysis of distributions, J. Functional Anal. (2) 146 (1997), 464-495.

[18] Y. V. Galperin, S. Samarah Time-frequency analysis on modulation spaces $M_{m}^{p, q}, 0<p, q \leq \infty$, Appl. Comput. Harmon. Anal. 16 (2004), 1-18.

[19] I. M. Gelfand, G. E. Shilov Generalized functions, I-III, Academic Press, NewYork London, 1968.

[20] ] M. A. de Gosson, Symplectic methods in harmonic analysis and in mathematical physics, Pseudo-Differential Operators Theory and Applications 7 Birkhäuser/Springer Basel AG, Basel, 2011.

[21] K. H. Gröchenig Describing functions: atomic decompositions versus frames, Monatsh. Math.112 (1991), 1-42.

[22] K. H. Gröchenig Foundations of Time-Frequency Analysis, Birkhäuser, Boston, 2001.

[23] K. Gröchenig, M. Leinert Wiener's lemma for twisted convolution and Gabor frames, J. Amer. Math. Soc. (1) 17 (2004), 1-18.

[24] K. Gröchenig,G. Zimmermann Spaces of test functions via the STFT J. Funct. Spaces Appl. 2 (2004), 25-53.

[25] L. Hörmander Pseudo-differential operators, Comm. Pure Appl. Math. 18 (1965), 501-517. 
[26] L. Hörmander The Analysis of Linear Partial Differential Operators, vol I, III, Springer-Verlag, Berlin Heidelberg NewYork Tokyo, 1983, 1985.

[27] J.J. Kohn and L. Nirenberg An algebra of pseudo-differential operators, Comm. Pure Appl. Math. 18 (1965), 269-305.

[28] C. Pfeuffer, J. Toft Compactness properties for modulation spaces, (preprint), arXiv:1804.00948.

[29] S. Pilipović Generalization of Zemanian spaces of generalized functions which have orthonormal series expansions, SIAM J. Math. Anal. 17 (1986), 477-484.

[30] S. Pilipović, N. Teofanov On a symbol class of Elliptic Pseudo-differential Operators, Bull. Acad. Serbe Sci. Arts 27 (2002), 57-68.

[31] S. Pilipović, N. Teofanov Pseudo-differential operators on ultra-modulation spaces, J. Funct. Anal.208 (2004), 194-228.

[32] A. D. Polyanin Handbook of Linear Partial Differential Equations for Engineers and Scientists, Chapman \& Hall/CRC Press, 2002.

[33] S. Rolewicz On a certain class of linear metric spaces, Bull. Acad. Polon. Sci. Sér. Sci. Math. Astrono. Phys., 5 (1957), 471-473.

[34] M. Ruzhansky, M. Sugimoto, N. Tomita, J. Toft Changes of variables in modulation and Wiener amalgam spaces, Math. Nachr. 284 (2011), 20782092.

[35] K. Tachizawa The boundedness of pseudo-differential operators on modulation spaces, Math. Nachr. 168 (1994), 263-277.

[36] N. Teofanov Ultramodulation spaces and pseudo-differential operators, Endowment Andrejević, Beograd, 2003.

[37] N. Teofanov Modulation spaces, Gelfand-Shilov spaces and pseudodifferential operators, Sampl. Theory Signal Image Process, 5 (2006), 225242.

[38] J. Toft Pseudo-differential operators with smooth symbols on modulation spaces, Cubo, 11 (2009), 87-107.

[39] J. Toft The Bargmann transform on modulation and Gelfand-Shilov spaces, with applications to Toeplitz and pseudo-differential operators, J. PseudoDiffer. Oper. Appl. 3 (2012), 145-227.

[40] J. Toft Gabor analysis for a broad class of quasi-Banach modulation spaces in: S. Pilipović, J. Toft (eds), Pseudo-differential operators, generalized functions, Operator Theory: Advances and Applications 245, Birkhäuser, 2015, 249-278.

[41] J. Toft Images of function and distribution spaces under the Bargmann transform, J. Pseudo-Differ. Oper. Appl. 8 (2017), 83-139.

[42] J. Toft Continuity and compactness for pseudo-differential operators with symbols in quasi-Banach spaces or Hörmander classes, Anal. Appl. 15 (2017), 353-389.

[43] J.Toft Continuity of Gevrey-Hörmander pseudo-differential operators on modulation spaces, (preprint) arXiv

Department of Mathematics, University of Turin, Italy

E-mail address: ahmed.abdeljawad@unito.it

Department of Mathematics, Linnæus University, Sweden

E-mail address: joachim.toft@lnu.se 